Journal of Mathematics and Statistics 1 (3): 217-224, 2005

ISSN 1549-3644

(C) 2005 Science Publications

\title{
Mathematical Models for the Dynamics of Tuberculosis in Density-dependent Populations: The Case of Internally Displaced Peoples' Camps (IDPCs) in Uganda
}

\author{
A. Ssematimba, J.Y.T. Mugisha and L.S. Luboobi \\ Department of Mathematics, Makerere University, P.O. Box 7062, Kampala, Uganda
}

\begin{abstract}
Mathematical models are formulated to establish the conditions (restrictions) on the size of the area occupied required minimizing and thereafter eradicating tuberculosis. Both numerical and qualitative analyses of the model are done and the effect of variation in the area size and recruitment rate on the different epidemiological groups is investigated. The results of the analysis show that there exists a stable disease-free equilibrium point provided that the characteristic area is greater than the product of the probability of survival from the latent stage to the infectious stage and the number of latent infections produced by a typical infectious individual during his/her mean infectious period. The study recommends that the characteristic area per individual should be at least 0.25 square kilometres in order to minimize the tuberculosis incidence.
\end{abstract}

Key words: Tuberculosis, density-dependence, IDPCs, characteristic area

\section{INTRODUCTION}

In 1993, concerned with the rising cases of deaths and infection rates, the World Health Organization (WHO) declared tuberculosis as a global emergency. Approximately a third of the worlds' population is affected by tuberculosis, particularly affecting people in developing countries where $99 \%$ of tuberculosis deaths occur. Of the 1.7 billion people estimated to be infected with tuberculosis, 1.3 billion live in developing countries. The resurgence of tuberculosis and other infectious diseases in the last decade has been closely linked with environmental and social changes that compromised people's immune systems and the social structures that are used to defend against diseases ${ }^{[1]}$. Developing and former communist countries will continue to experience the greatest impact from infectious diseases because of malnutrition, poor sanitation, poor water quality and inadequate health care but developed countries also will be affected ${ }^{[2]}$.

Many epidemiological studies in the past have already proven the direct relationship of tuberculosis with poverty and underdevelopment. Tuberculosis occurs in the poorest and most underdeveloped countries of the world and within societies, it occurs in the most socioeconomically deprived sectors, where factors contributing to its spread exist in overcrowding, malnutrition and lack of access to health care services. Progression rates to active tuberculosis correlate with socioeconomic measures. An increase in the number of people per room or alternative measures of overcrowding increase the chances of infection by prolonged exposure.

The unresolved political conflicts in developing world have led to persistent wars which have forced people into safety camps, as refugees or as internally displaced people. Most of these camps are congested/overcrowded with extremely poor sanitation. Cold, sleeping rough and in the bush all lead to an increased incidence of respiratory ailments of all kinds. Overcrowding and poor hygiene ensure a rapid transmission of these diseases within the family units and treatment of tuberculosis, which takes approximately eight months is quite costly in areas far from hospitals ${ }^{[3]}$. In camps, access to health care and the quality of service is lower than in other areas and as a result, preventative care is less available and the treatment of tuberculosis is less extensive and effective and because of this, some cases go untreated and those affected often die.

In Uganda, tuberculosis cases are more in urban concentrations and other densely populated areas. The distribution of tuberculosis cases by district in 1999 indicated that Uganda had a total of 29,994 tuberculosis cases of which the leading were Kampala with 5,525, 1,371 in Mbarara, 1,325 in Gulu, 1,323 in Lira and the least were Kalangala with only 48 and Ssembabule with only $54^{[4]}$. The high levels of the tuberculosis incidence in Northern Uganda (Gulu and Lira) is as a result of overcrowding in the IDPCs and lack of adequate housing in rural areas abandoned as a result of war.

As of July 2004, the population figures in these camps were as follows; Pabbo camp, the biggest of all the camps in Gulu district harbored 55,043 people, Acet camp with 24,876 people, Amuru camp with 39,877 people, Anaka camp with 27,972 people, Atiak camp with 23,655 people, Awere camp with 21,434 people Opit camp with 25,682 people $^{[5]}$. There is very limited space in these camps for human activities and the make-shift structures that people occupy are not

Corresponding Author: J.Y.T. Mugisha, Faculty of Computing and Information Technology, Makerere University, P.O. Box 7062, Kampala, Uganda 
properly ventilated to ensure proper sanitation. Displaced people are at a greater risk of becoming infected with tuberculosis due to the close contact with the infectious individuals.

With the many political conflicts in the developing countries, the aspect of high population density in settlements and the health risks associated with overcrowding such as poor sanitation and high level of disease incidences have become rampant. In this study, we investigate the effect of population density on the dynamics of tuberculosis.

The Model: Now, using the mechanics of transmission as in the model of ${ }^{[6]}$, we formulate mathematical models that incorporate a parameter for the size of the area occupied by the population being studied in the transmission term so as to help analysis the densitydependence of the dynamics of tuberculosis. In our study, we assume that there is a homogeneous mixing of the population where all people are equally likely to be infected by the infectious individuals in case of contact, the population is distributed over the whole settlement (in the specified area) with a negligibly small area, compared to the camp size, spared for other human activities and we further assume that all immigrants and newborns are uninfected and therefore join the susceptible category.

Variables and parameters: In the density-dependent model for the dynamics of tuberculosis, we use the following variables and parameters:

$S(t)$ : the number of susceptible individuals at time, $t$

$L(t)$ : the number of latently infected/exposed individuals at time $t$

$I(t)$ : the number of infectious individuals at time $t$

$T(t)$ : the number of recovered/treated individuals at time $t$

A: the total area of the camp occupied by the population

$\Lambda: \quad$ Recruitment rate

$\mu$ : the per capita natural mortality rate

$d: \quad$ the tuberculosis-induced mortality rate

$\beta_{1}$ : the probability that a susceptible individual becomes infected by one infectious individual per contact per unit time

$\beta_{2}$ : the probability that a treated individual becomes infected by one infectious individual per contact per unit time

$k$ : the rate of progression to active tuberculosis

$r_{1}: \quad$ recovery rate of the latent class

$r_{2}: \quad$ recovery rate of the infectious class

$c$ : the per capita contact rate

The dynamics of a one-strain model of tuberculosis can be described as in the compartmental model Fig. 1.

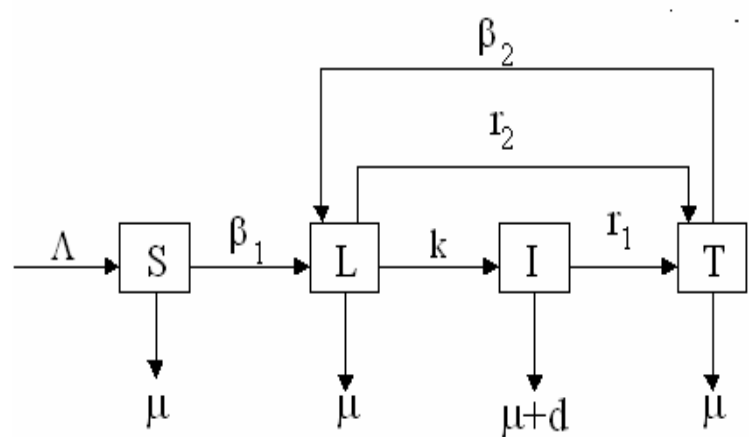

Fig. 1: A compartmental diagram for a one-strain model of tuberculosis

Equations of the model: Applying the assumptions and the inter-relations between the variables and parameters as described in the compartmental model in Fig. 1, the density dependent dynamics of tuberculosis are described by the following equations:

$$
\begin{aligned}
& \frac{d S}{d t}=\Lambda-\mu S-\beta_{1} c S \frac{I}{A} \\
& \frac{d L}{d t}=\beta_{1} c S \frac{I}{A}-\left(\mu+k+r_{1}\right) L+\beta_{2} c T \frac{I}{A} \\
& \frac{d I}{d t}=k L-\left(\mu+d+r_{2}\right) I \\
& \frac{d T}{d t}=r_{1} L+r_{2} I-\mu T-\beta_{2} c T \frac{I}{A}
\end{aligned}
$$

where $N=S+L+I+T$ is the total population size.

\section{ANALYSIS OF THE MODEL}

Existence of steady states: Let $E\left(S^{*}, L^{*}, I^{*}, T^{*}\right)$ be the equilibrium points of the system described by the equations (2.1) - (2.4). At an equilibrium point, we have $\frac{d S}{d t}=\frac{d L}{d t}=\frac{d I}{d t}=\frac{d T}{d t}=0$

a. The existence of the trivial equilibrium point: For as long as the recruitment term, $\Lambda$ is not zero; the population will not be extinct. This implies that there is no trivial equilibrium point i.e. $\left(S^{*}, L^{*}, I^{*}, T^{*}\right) \neq(0,0,0,0)$.

b. The existence of the disease-free equilibrium point, $\left(E_{0}\right)$ : At the disease free equilibrium, we have $I^{*}, L^{*}=0$. This gives $E_{0}=\left(\frac{\Lambda}{\mu}, 0,0,0\right)$. We define $S_{0}=\frac{\Lambda}{\mu}$ as the asymptotic carrying capacity of the population. We now investigate the stability of the 
disease-free equilibrium point, $E_{0}=\left(\frac{\Lambda}{\mu}, 0,0,0\right)$. The Jacobian matrix at $E_{0}$ is given by

$$
J_{E_{0}}=\left(\begin{array}{cccc}
-\mu & 0 & -\beta_{1} c\left(\frac{\Lambda / \mu}{A}\right) & 0 \\
0 & -\left(\mu+k+r_{1}\right) & \beta_{1} c\left(\frac{\Lambda / \mu}{A}\right) & 0 \\
0 & k & -\left(\mu+d+r_{2}\right) & 0 \\
0 & r_{1} & r_{2} & -\mu
\end{array}\right)
$$

If $\lambda_{i}$ are the eigenvalues of $J E 0$, then

$(\lambda+\mu)^{2} \operatorname{det}\left(\begin{array}{cc}-\left(\mu+k+r_{1}\right)-\lambda & \beta_{1} c\left(\frac{\Lambda / \mu}{A}\right) \\ k & -\left(\mu+d+r_{2}\right)-\lambda\end{array}\right)=0$
Let $B=\left(\begin{array}{cc}-\left(\mu+k+r_{1}\right) & \beta_{1} c\left(\frac{\Lambda / \mu}{A}\right) \\ k & -\left(\mu+d+r_{2}\right)\end{array}\right)$. Then, from matrix

$B$

we

have

$\operatorname{det}(B)=\left(\mu+k+r_{1}\right)\left(\mu+d+r_{2}\right)-k \beta_{1} c\left(\frac{\Lambda / \mu}{A}\right)$ and

$\operatorname{Trace}(B)=-(\mu+k+r 1)-(\mu+d+r 2)$. Clearly, Trace $(B)<0$ since all the parameters $\mu, d, r 1, r 2, k, \beta_{1} c$ and $A$ are positive.

For the determinant of $B$ to be $>0$, we should have $\left(\frac{\Lambda / \mu}{A}\right)<\left(\frac{\mu+k+r_{1}}{k}\right)\left(\frac{\mu+d+r_{2}}{\beta_{1} c}\right)$ Therefore, the disease-free equilibrium point, $E_{0}$ is stable provided

$\left(\frac{A}{\Lambda / \mu}\right)>\left(\frac{k}{\mu+k+r_{1}}\right)\left(\frac{\beta_{1} c}{\mu+d+r_{2}}\right)$

where

$\left(\frac{A}{\Lambda / \mu}\right)$ is the area occupied per individual also referred to as characteristic area.

$\left(\frac{k}{\mu+k+r_{1}}\right)$ is the probability of survival from latent stage into the infectious stage.

$\left(\frac{\beta_{1} c}{\mu+d+r_{2}}\right)$ is the number of latent infections produced by a typical infectious individual during his/her mean infectious period.

Thus, to have a Tuberculosis-free stable population, the characteristic area per unit individual must always be greater than the product of the probability of survival from latent stage into the infectious stage and the number of latent infections produced by a typical infectious individual during his/her mean infectious period. The term, $\left(\frac{k}{\mu+k+r_{1}}\right)\left(\frac{\beta_{1} c}{\mu+d+r_{2}}\right)$ defines the critical area size required for eradication of tuberculosis.

c. The possibility of existence and stability of the endemic equilibrium point: Let $E_{1}=\left(S^{*}, L^{*}, I^{*}, T^{*}\right)$ be the endemic equilibrium point, where $\left(S^{*}, L^{*}, I^{*}, T^{*}\right) \neq 0$. At the endemic equilibrium point, $E_{1}$, the Jacobian matrix becomes

$$
J_{E_{1}}=\left(\begin{array}{cccc}
-\left(\mu+\beta_{1} c\left(\frac{I^{*}}{A}\right)\right. & 0 & -\beta_{1} c\left(\frac{S^{*}}{A}\right) & 0 \\
\beta_{1} c\left(\frac{I^{*}}{A}\right) & -\left(\mu+k+r_{1}\right) & \beta_{1} c\left(\frac{S^{*}}{A}\right)+\beta_{2} c\left(\frac{T^{*}}{A}\right) & \beta_{2} c\left(\frac{I^{*}}{A}\right) \\
0 & k & -\left(\mu+d+r_{2}\right) & 0 \\
0 & r_{1} & r_{2}-\beta_{2} c\left(\frac{T^{*}}{A}\right) & -\left(\mu+\beta_{2} c\left(\frac{I^{*}}{A}\right)\right.
\end{array}\right)
$$

This approach renders the conclusion impossible since the computations become complex. Feng et al. ${ }^{[7]}$ described the use of basic reproductive number, $R_{0}$ in analyzing stability of the endemic equilibrium point. According to their work, when $R_{0}>1$ the system has a unique endemic equilibrium that is globally asymptotically stable. We also adopt the same technique in the section that discusses the basic reproductive number.

Conditions for the minimization of the disease: For the minimization of the disease incidence, we require that the population sizes of the latently infected and the infectious individuals decrease. This is attained when $\frac{d L}{d t}<0$ and $\frac{d I}{d t}<0$ from which we obtain $\beta_{1} c S \frac{I}{A}-\left(\mu+k+r_{1}\right) L+\beta_{2} c T \frac{I}{A}<0$ and $k L-\left(\mu+d+r_{2}\right) I<0$. Combining these gives $A>\left\{\frac{k}{\mu+k+r_{1}}\right\}\left\{\frac{\beta_{1} c S+\beta_{2} c T}{\mu+d+r_{2}}\right\}$.

Therefore, in order to minimize the incidence of tuberculosis in density-dependent populations the area occupied, $A$ must always be greater in magnitude than the product of the probability of survival from latent stage into the infectious stage, $\left\{\frac{k}{\mu+k+r_{1}}\right\}$ and the number of latent infections produced by a typical infectious individual during his/her mean infectious period, $\left\{\frac{\beta_{1} c S+\beta_{2} c T}{\mu+d+r_{2}}\right\}$.

Expected populations after eradication of the disease: Eradication of the disease from the community 
occurs when there are no more exposed and infective individuals in the population. This is when $I=L=0$ and in this case, the total population is $N=S+T$. Equations (2.1) and (2.4) reduce to $\frac{d S}{d t}=\Lambda-\mu S$ and $\frac{d T}{d t}=-\mu T$, respectively. Solving these equations gives $S(t)=\frac{\Lambda}{\mu}+\left(S_{i}-\frac{\Lambda}{\mu}\right) e^{-\mu t} \quad$ and $\quad T=T_{i} e^{-\mu t}, \quad$ where $S_{i}$ and $T_{i}$ are the initial number of susceptible and treated individuals respectively. As $t \rightarrow \infty$, the population size of the susceptibles, $S(t) \approx \frac{\Lambda}{\mu}$, where $\frac{\Lambda}{\mu}$ is the asymptotic carrying capacity of the total population. Also, $T(t) \rightarrow 0$ whenever $t \rightarrow \infty$ and hence the whole population will be comprising of only susceptibles.

The basic reproductive number, $\boldsymbol{R}_{\boldsymbol{0}}$ : Diekmann et $a l^{[8,9]}$, defined the basic reproductive number, $R 0$ as the average number of secondary infections caused by an infectious individual during his/her entire life as an infectious person.

Tuberculosis infection and re-infection are always existent in a community due to respiratory contact between the susceptibles individuals, treated individuals and the infectious individuals. Whether the disease becomes persistent or dies out depends on the magnitude of the basic reproductive number, $R_{0}$. Stability of equilibrium points can be analyzed using $R_{0}$. The disease-free equilibrium is locally asymptotically stable if $R_{0}<1$ and is unstable if $R_{0}>1$. In other words, when $R_{0}<1$, every infectious individual will cause less than one secondary infection and hence the disease will die out and when $R_{0}>1$, every infectious individual will cause more than one secondary infection and hence an epidemic. All public health control measures are usually based on methods that, if effective, would lower $R_{0}$ to below unity.

We derive the expression for $R 0$ using the next generation operator approach ${ }^{[8,9]}$. In this approach, the population is categorized into three classes and for the density-dependent model in this study, the three classes are $X=(S, T), Y=(L)$ and $Z=(I)$ and the disease-free equilibrium is $U_{0}=\left(\frac{\Lambda}{\mu}, 0,0\right)$.

From the equations of the model, define

$$
\begin{aligned}
& \frac{d X}{d t}=\Lambda-2 \mu X-\beta_{1} c X\left(\frac{Z}{A}\right)+r_{1} Y+r_{2} Z-\beta_{2} c X\left(\frac{Z}{A}\right)=f(X, Y, Z) \\
& \frac{d Y}{d t}=\beta_{1} c X\left(\frac{Z}{A}\right)-\left(\mu+k+r_{1}\right) Y+\beta_{2} c X\left(\frac{Z}{A}\right)=g(X, Y, Z)
\end{aligned}
$$

$\frac{d Z}{d t}=k Y-\left(\mu+d+r_{2}\right) Z=h(X, Y, Z)$

Comparing our functions with those used in the next generation operator approach ${ }^{[8,9]}$ gives $g\left(X^{*}, Y, Z\right)=\beta_{1} c\left(\frac{\Lambda}{\mu}\right)\left(\frac{Z}{A}\right)-\left(\mu+k+r_{1}\right) Y+\beta_{2} c\left(\frac{\Lambda}{\mu}\right)\left(\frac{Z}{A}\right)=0$ $g\left(X^{*}, Y, Z\right)=0$ is assumed to implicitly determine a function $Y=\hat{g}\left(X^{*}, Y\right)$, and this yields

$$
Y=\frac{\left(\beta_{1}+\beta_{2}\right) c\left(\frac{\Lambda}{\mu}\right)\left(\frac{Z}{A}\right)}{\left(\mu+k+r_{1}\right)} .
$$

The function, $h\left(X^{*}, \hat{g}\left(X^{*}, Y\right), Z\right)$ is obtained as $h\left(X^{*}, \hat{g}\left(X^{*}, Y\right), Z\right)=\frac{k\left(\beta_{1}+\beta_{2}\right) c\left(\frac{\Lambda}{\mu}\right)\left(\frac{Z}{A}\right)}{\left(\mu+k+r_{1}\right)}-\left(\mu+d+r_{2}\right) Z$

Next is to obtain the partial derivative with respect to $\mathrm{Z}$ of $h\left(X^{*}, \hat{g}\left(X^{*}, 0\right), 0\right)$ and thereafter let the matrix $H=\frac{\partial h}{\partial Z}$. This gives

$$
H=\frac{k\left(\beta_{1}+\beta_{2}\right) c\left(\frac{\Lambda / \mu}{A}\right)}{\left(\mu+k+r_{1}\right)}-\left(\mu+d+r_{2}\right)
$$

Letting $H=M-D$ with $M \geq 0$ and $D>0$, a diagonal matrix, we obtain

$M=\frac{k\left(\beta_{1}+\beta_{2}\right) c\left(\frac{\Lambda / \mu}{A}\right)}{\left(\mu+k+r_{1}\right)}$ and $D=\left(\mu+d+r_{2}\right)$

From the above expressions, the basic reproductive number, $R_{0}$, defined as the spectral radius (dominant eigenvalue) of the matrix $M D^{-1}$, $R_{0}=\rho\left(M D^{-1}\right)^{[8,9]}$, is given by

$R_{0}=\left(\frac{\Lambda / \mu}{A}\right)\left(\frac{\left(\beta_{1}+\beta_{2}\right) c}{\mu+d+r_{2}}\right)\left(\frac{k}{\mu+k+r_{1}}\right)$

Where, $\left(\frac{\Lambda / \mu}{A}\right)$ is the density of the susceptible population $\beta_{1} c$ and $\beta_{2} c$ are the effective transmission rates $\left(\frac{1}{\mu+d+r_{2}}\right)$ is the effective infectious period $\left(\frac{\left(\beta_{1}+\beta_{2}\right) c}{\mu+d+r_{2}}\right)$ is the number of latent infections produced by a typical infectious individual during the mean infectious period $\left(\frac{k}{\mu+k+r_{1}}\right)$ is the probability of survival from latent stage into the infectious stage 
Note that if $R_{0}<1$, the disease-free equilibrium point is globally asymptotically stable while if $R_{0}>1$, the disease-free equilibrium is unstable and the endemic equilibrium is globally asymptotically stable ${ }^{[7]}$. Thus;

a. When $R_{0}<1$, we have $\left(\frac{\lambda \mu}{\mathrm{A}}\right)\left(\frac{\left(\beta_{1}+\beta_{2}\right) \mathrm{c}}{\mu+\mathrm{d}+\mathrm{r}_{2}}\right)\left(\frac{\mathrm{k}}{\mu+\mathrm{k}+\mathrm{r}_{1}}\right)<1$, implying that $\left(\frac{\mathrm{A}}{\mathrm{A}_{\mu}}\right)>\left(\frac{\left(\beta_{1}+\beta_{2}\right) \mathrm{c}}{\mu+\mathrm{d}+\mathrm{r}_{2}}\right)\left(\frac{\mathrm{k}}{\mu+\mathrm{k}+\mathrm{r}_{1}}\right)$

b. When $R_{0}>1$, we have $\left(\frac{A_{\mu}}{\mathrm{A}}\right)>\left(\frac{\left(\beta_{1}+\beta_{2}\right) \mathrm{c}}{\mu+\mathrm{d}+\mathrm{r}_{2}}\right)\left(\frac{\mathrm{k}}{\mu+\mathrm{k}+\mathrm{r}_{1}}\right)$

The density of the susceptibles, $\left(\frac{\Lambda / \mu}{A}\right)$ emphasizes the role played by the size of the area, $A$ occupied by the population. When the area is big enough, the density will be small thereby reducing on the size of the basic reproductive number. And when the area size $A$ is small, the density will be higher and consequently the basic reproductive number or the number of secondary infections due to a single infectious individual will be higher.

Numerical simulation of the model: Below are some of the parameter values used:

a. Natural mortality rate of individuals, $(\mu)$ : The time unit is set at year and the constant natural mortality rate, $\mu$ is assumed to be inversely related to life expectancy at birth which is approximately 45 years in Uganda.

$\mu=\frac{1}{45}=0.0222$ per year

b. Recruitment rate, $(\Lambda)$ : The recruitment rate, $(\Lambda)$ controls the total population sizes because the asymptotic carrying capacity of the population is $\frac{\Lambda}{\mu}$. This is set at $\frac{\Lambda}{\mu} \approx 10^{6}[10]$. For purposes of this study, we shall set the recruitment rate at 1,500 individuals per year.

c. Per-capita Recovery rate, $(r)$ : This is taken as the inverse of the time between tuberculosis activation and recovery by treatment. According to Styblo ${ }^{[11]}$ it is between 4 and 6 months and according to Feng et al. ${ }^{[7]}$, the treatment period is about 6 to 9 months implying that $r_{1}>1$ per year. Taking the treatment period to be 8 months, we obtain the recovery rate as $\frac{1}{0.5}=2$ per individual per year. According to ${ }^{[11]}$, recovery rate $\frac{1}{0.67}=1.5$ per individual per year. For this study, given the minimal difference between the recovery rates of latently infected and infectious individuals, we shall set both $r_{1}$ and $r_{2}$ at 1.5 per individual per year.

d. Per capita rate of infection, $(\beta)$ and contact rate $(c)$ : This is set to match the expected number of infections produced in $\frac{1}{\mu+d+r_{2}}$ units of time. The mean effective infectious period, $\frac{1}{\mu+d+r_{2}}$ is less than the period of time during which the individual is potentially infectious. In fact, most of the infections produced by an infectious individual occurred during the first months of infectiousness ${ }^{[12]}$. According to Aparicio et al. ${ }^{[10]}$, in big cities the number of contacts per day increases significantly. For example, individuals who rely on mass transportation (overcrowded conditions) may experience around 100 close contacts per day (in a poorly ventilated place). Therefore, in the case of IDPCs, we can take up 100 effective contacts per year. Song et al..$^{[13]}$ suggested $\beta$ to be 2 . We also consider $\beta_{1}=\beta_{2}$ since the difference is negligible.

e. Per capita disease-induced mortality rate, $(d)$ : The disease-induced mortality rate, $d$ varies from country to country. It is as low as 0.07 in developed countries but reaches 0.365 per year in some African countries (Snider et al[6,14]. For Uganda therefore, we take $d=0.365$

f. Average per-capita Progression rate to active tuberculosis, $(k)$ : Latent periods range from months to decades. Most infected individuals never progress towards the active tuberculosis state. On the other hand, average infectious periods are relatively short (few months) and becoming shorter in developing nations due to the availability of treatment ${ }^{[13]}$. According to Aparicio et al. ${ }^{[10]}$, the average per-capita rate of progression to active tuberculosis is estimated from $x=\left(\frac{k}{\mu+k+r_{1}}\right)$, that is, from the proportion of infected individuals that develops active tuberculosis during his/her lifespan. $x$ is estimated to be between 5\% and $10 \%$ in developed countries and obviously higher in developing countries. If the life expectancy is 45 years and hence $\mu=0.0222$, for the case of Uganda and if $15 \%$ of the latent population eventually develops active tuberculosis, then $k$ is computed as: $k=\frac{x\left(\mu+r_{1}\right)}{1-x}=0.00396$ year $^{-1}$ 
(a) Susceptibles, S with changing A rea size, A

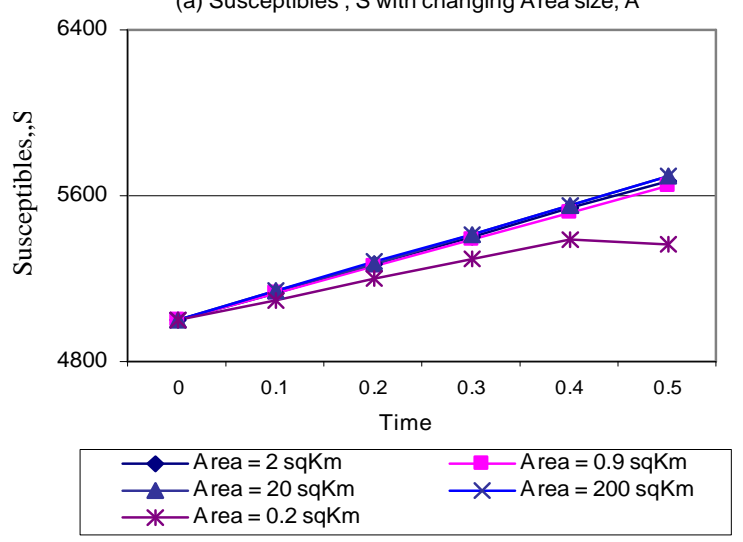

(c) Infecteds, I with changing Area size, A

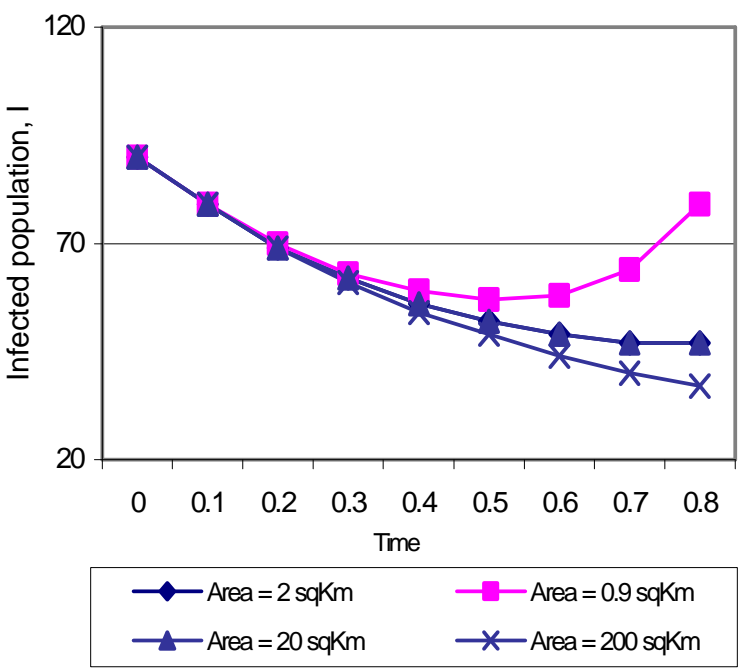

(b) Exposed/Latent, $L$ with changing Area, $A$

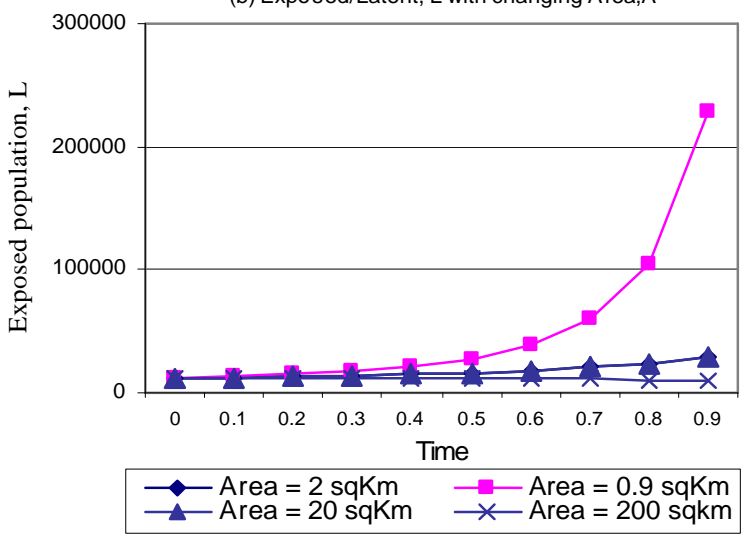

(d) Treated Population, T with changing Area size, A

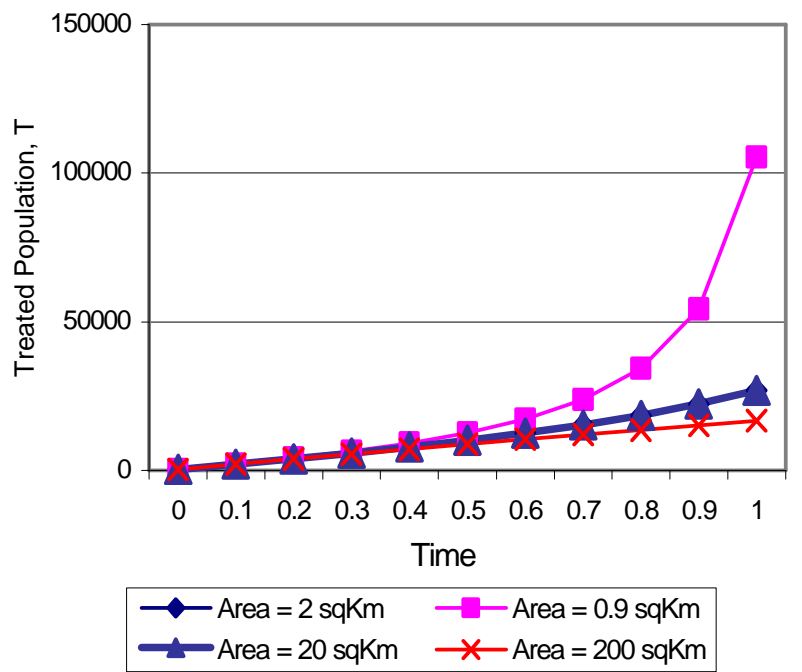

Fig. 2: The effect of size of the area occupied, $A$ on the sizes of the different epidemiological classes

The effect of variation of the size of the area occupied, $A$ on the different epidemiological classes: This effect is studied using $A=0.2$ square kilometres, 0.9 square kilometres, 2 square kilometres, 20 square kilometres, 200 square kilometres. We observe in Fig. 2a that when the size of the area occupied, $A$ is reduced from 2 square kilometres to 0.2 square kilometres thereby increasing the population density, the population will first increase because of the recruitment rate, ? through birth and immigration. It will there after decline because of the increased disease incidence. There are more infections resulting from the close contact due to high population density. When the area is increased to say 20 square kilometres or 200 square kilometres, there is a slight deviation in the population sizes in the two cases, implying that there is a threshold area size. However, in both cases, the number of susceptibles will increase because of the reduced disease incidence and recruitment.

Figure $2 \mathrm{~b}$ shows that for a smaller area size, $A=$ 0.9 square kilometres hence higher population density, the increase in population size of the latently infected individuals shall be faster than in all the other cases. This is as a result of increased infection rate due to a higher contact rate of the susceptibles with the infectious individuals. We further observe that with lower population density (when the area is bigger say, $A=20$ square kilometres or 200 square kilometres), the number of latent individuals increases, though slowly.

It is observed in Fig. 2c that irrespective of the area size, the number of infectious individuals declines in a small time interval, though at different rates. After a while, we observe that the population increases in all the cases, with a higher and faster increase when the population density is higher. It is further clearly observed that, at some point when for other area sizes the number of infecteds is declining, it is on the rise when the area is so small. This is because in a congested community, there is a higher rate of infection and hence a big number of susceptibles becomes infected and progress to infectious stage.

A delay in the increase of the population size of treated individuals is observed in Fig. $2 \mathrm{~d}$ when the area is bigger $(A=200$ square kilometres and 20 square kilometres). However, an increase is observed in all the cases. These increments occur in the period when the number of infecteds is declining as observed in the first phases. 
(a) Susceptibles, S with changing Recruitment rate

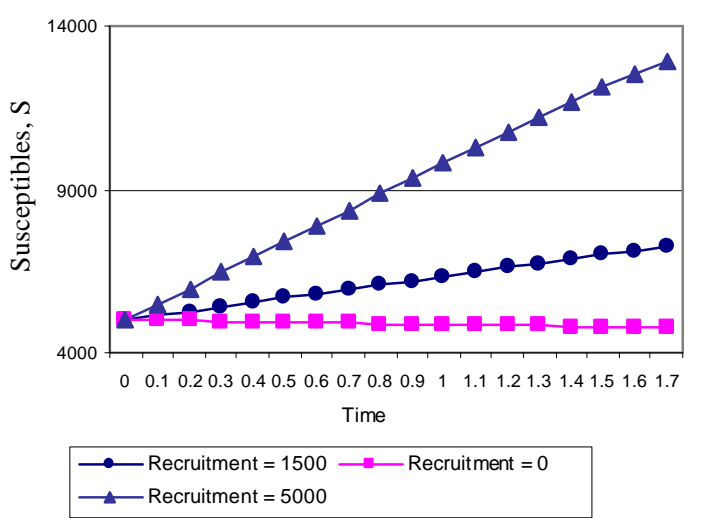

(c) Infecteds, I with changing Recruitment rate

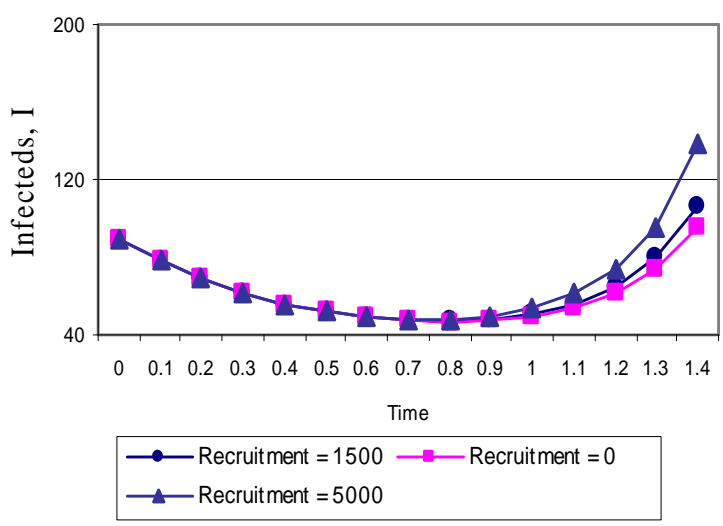

(b) Exposed, L with changing Recruitment rate

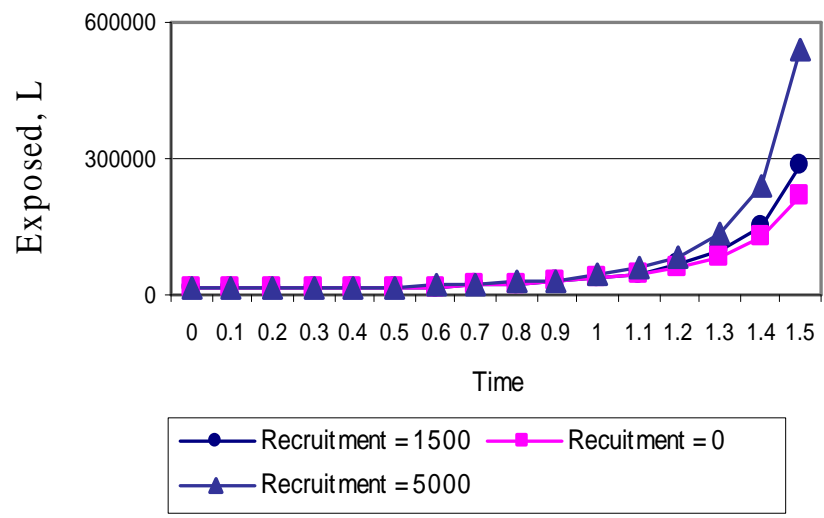

(d) Treated, T with changing Recruitment rate

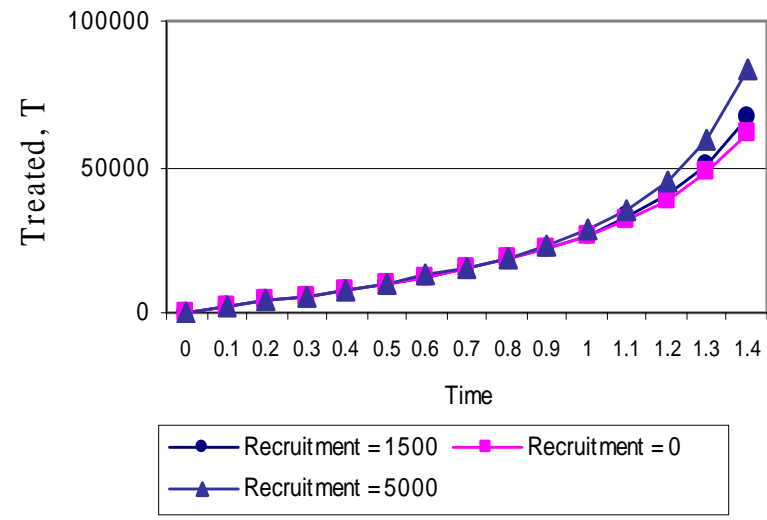

Fig. 3: The effect of recruitment rate, $\Lambda$ on the sizes of the different epidemiological classes

The effect of variation of the recruitment rate, $\Lambda$ on the different epidemiological classes: The above effect is studied with the recruitment rate, $\Lambda=0,1500$, 5000 individuals. Figure 3 shows the effect of varying the rate of recruitment into the population. The number of susceptibles is seen to decline (Fig. 3a) when there is totally no recruitment into the system i.e. $\Lambda=0$. Since recruitment in this case entails immigration and/or birth, it is expected that without any recruitment, the population of susceptibles would be constant. But in this case, they are dying naturally at a rate $\mu$ and some are being infected. Therefore, without any recruitment, the population of susceptibles will be extinct. However, when the recruitment rate is more than tripled to 5000 from 1500, the population is observed to grow faster.

In Fig. 3b, the population size of latently infected individuals is seen to first grow steadily at a small rate and thereafter increase steeply. The time at which the population explodes or grows greatly varies according to the recruitment rate. With recruitment rate at 5000 individuals, the population grows faster compared to the original recruitment rate of 1500 . This is because, with a very high recruitment rate, the susceptibles become many and the congestion, which in turns results into infection due to increased contact rate, is increased. Further still, we see that there is a bigger delay when there is no recruitment into the system.
This is as a result of the decreased number of susceptibles who contribute partially to the latent class when they are infected through contact with infectious individuals.

The population size of the infectious individuals is observed in Fig. 3c to first decline in the initial stages for all recruitment rates but later increase steeply. This increment is exactly in accordance with the available number of exposed individuals. A similar trend is observed with the population size of the treated individuals as evidenced in Fig. 3d.

\section{RESULTS AND DISCUSSION}

In the search for the possible ways of eradicating tuberculosis, there is need to address the issue of limited knowledge on the biology of tuberculosis (mechanisms of transmission, life cycle of the transmitting vector and effectiveness of the existing drugs in fighting this vector) and the host-parasite relationship. In Uganda, the disease incidence is on the increase as per the available records ${ }^{[15]}$ and among others, overcrowding and unsanitary conditions play a major role in explaining the observed trends in Uganda and the world over. In order to control the tuberculosis incidence, these issues must be addressed. 
In this research, the effect of population density on the dynamics of tuberculosis is discussed. It should be noted that population density determines the level of respiratory contact in a community and this level directly determines the infection rate of airborne diseases like tuberculosis. Overcrowding is most in urban areas and now settlement camps such as internally displaced peoples' camps and refugee camps. In order to carry out the investigations, a mathematical model was formulated that incorporated the size of the area occupied by the population being studied and areas with very high population densities were chosen for estimation of parameters. The results suggested that the observed trend in tuberculosis incidence was partly as a result of explosive population growth due to urbanization and resettlements (for the case of IDPCs).

In the qualitative analysis of the model, the existence of steady states and their stabilities were analyzed. The analysis showed that a disease-free equilibrium point existed and was found to be globally asymptotically stable provided the characteristic area per individual is greater than the product of the probability of survival from latent stage into the infectious stage and the number of latent infections produced by a typical infectious individual during the mean infectious period and it is the same requirement for minimization of the disease incidence. Basing on our findings, if tuberculosis is to be eradicated, it is recommended that the characteristic area per individual to be 0.25 square kilometres.

The results of numerical simulation where remarkably in line with those of the qualitative analysis of the model in that, they both emphasized the dependence of tuberculosis incidence on the level population density in the community hence the area occupied by the population under study. Increments in the magnitude of the recruitment rate were observed to come hand in hand with an increased disease incidence and this is because of the increase in the level of contact due to a higher population density.

The basic reproductive number, $R_{0}$ is one of the tools used in determining disease trends ${ }^{[8]}$. The necessary condition for $R 0$ being less than unity, in which case there would be no more tuberculosis epidemics, is the same as the requirement for the stability of the disease-free equilibrium point. The size of the area occupied is so significant in determining the basic reproductive number in our case. The necessary condition to have $R_{0}<1$ clearly stipulates the role played by population density in determining the disease trends in a community. The higher the density, the greater the risk of instability of the disease-free equilibrium, implying that there is a possibility of an epidemic in the community.

\section{ACKNOWLEDGEMENT}

The authors would like to thank the management committee of Gulu University and NUFU for the financial support.

\section{REFERENCES}

1. Miranda, D., 2003. Tuberculosis: Facts, challenges and courses of action. Health Alert Asia-Pacific Edn., HAIN- Health Action Information Network, 2.

2. Gannon, J.C., 2000. The global infectious disease threat and its implications for the United States. Central Intelligence Agency, pp: 1-39.

3. Dorsey, J. and S. Opeitum, 2002. The net economic cost of the conflict in the Acholiland sub region of Uganda: Civil Society Organization for Peace in Northern Uganda (CSOPNU), pp: 1-40.

4. National Tuberculosis and Leprosy Programme, 1999. Ministry of Health, Uganda Status reports.

5. World Food Programme and Norwegian Refugee Council Report, 2004.

6. Song, B., C. Castillo-Chavez and J.P. Aparicio, 2002. Tuberculosis models with fast and slow dynamics: The role of close and casual contacts. Math. Biosci., 180: 187-205.

7. Feng, Z., W. Huang and C. Castillo-Chavez, 2001. On the role of variable latent periods in mathematical models for tuberculosis. J. Dynamics and Differential Equations, 13: 425-452.

8. Diekmann, O., J.A. Heesterbeek and J.A.J. Metz, 1990. On the definition and the computation of the basic reproductive ratio, $\mathrm{R} 0$ in models of infectious diseases in heterogeneous populations. J. Math. Biol., 28: 365-382.

9. Castillo-Chavez, C., Z. Feng and W. Huang, 2002. On the computation Ro and its role on global stability. In: Castillo-Chavez, C., Blower, S., van den Driesschie, Kirschner, D. and Yakubu, A.A. (Eds.). Mathematical Approaches for Emerging and Reemerging Infectious Diseases: An Introduction, IMA Volumes in Mathematics and its Applications, Springer, 125: 229-250.

10. Aparicio, J.P., A.F. Capurro and C. CastilloChavez, 2000. Transmission and dynamics of tuberculosis on generalized households. J. Theor. Biol., 206: 327-341.

11. Styblo, K., 1991. Selected papers, epidemiology of tuberculosis. Royal Netherlands Tuberculosis Assoc., 24: 55-62.

12. Daniel, T.M., 1997. Captain of Death: The Story of tuberculosis. NY: University of Rochester Press, pp: 37-39.

13. Song, B., C. Castillo-Chavez and J.P. Aparicio, 2002. Global dynamics of tuberculosis models with density dependent demography. In: Mathematical Approaches for Emerging and Reemerging Infectious Diseases. IMA J. Math. Appl. Med. Biol., 126: 275-294.

14. Snider, D.E., M. Raviglione and A. Kochi, 1994. Global burden of tuberculosis: Tuberculosis Pathogenesis and Control Bloom, B. Ed. WA, U.S.A., ASM.

15. World Health Organization, 2003. Tuberculosis Fact Sheet: Global Tuberculosis Program, Geneva. 